\title{
Application of penalty methods to generalized variational inequalities in Banach spaces
}

\author{
G. W. Su' ${ }^{\mathrm{a}}$ Z. W. Zhao
}

${ }^{a}$ Guangxi Key Laboratory Cultivation Base of Cross-border E-commerce Intelligent Information Processing, College of Information and Statistics, Guangxi University of Finance and Economics, Nanning, Guangxi, 530003, P. R. China.

${ }^{b}$ Guangxi Key Laboratory of Universities Optimization Control and Engineering Calculation, and College of Sciences, Guangxi University for Nationalities, Nanning, Guangxi 530006, P. R. China.

Communicated by Y.-B. Xiao

\begin{abstract}
In this paper, we consider a class of generalized variational inequalities (GVI) in infinite dimensional Banach spaces, in which only approximation sequences for GVI are known instead of exact values of the cost mapping and feasible set. A sequence of inexact solutions of auxiliary problems involving general penalty method is introduced. We obtain some convergence properties of the perturbed version of the regularized penalty method under mild coercive conditions, which extend some well-known results of variational inequalities in many respects. (C)2017 All rights reserved.
\end{abstract}

Keywords: Generalized variational inequality, penalty method, regularization, coercivity conditions, equilibrium problem. 2010 MSC: 47J22, 34A60.

\section{Introduction}

Variational inequality theory, introduced in the early 1960s, has played a critical and significant role in nonlinear analysis. This field has witnessed an explosive growth in both theory and applications. Recently, research on non-stationary generalized variational inequalities has attracted the attention of a considerable number of scholars.

Let $X$ be a nonempty subset of a Banach space $E$ and $G: X \rightarrow 2^{E^{*}}$ be a set-valued mapping. In this work, we consider the following generalized variational inequality: find an element $x^{*} \in X$ and $g^{*} \in G\left(x^{*}\right)$ such that

$$
\left\langle\mathrm{g}^{*}, y-\mathrm{x}^{*}\right\rangle \geqslant 0 \text { for all } \mathrm{y} \in \mathrm{X}
$$

Particularly, if $G$ is a single-valued mapping, then GVI (1.1) reduces to the classical variational inequality (VI, for short): find an element $x^{*} \in X$ such that

$$
\left\langle G\left(x^{*}\right), y-x^{*}\right\rangle \geqslant 0 \text { for all } y \in X .
$$

\footnotetext{
*Corresponding author

Email addresses: suguangwang@163.com (G. W. Su), zhengweizhao100@126.com (Z. W. Zhao)
} 
GVIs arise in economics, mathematical physics, and other general problems in nonlinear analysis, such as optimization, fixed point, game equilibrium, and complementarity problems, see, for example, $[3,7,9,10,17-25,29,30,32]$ and references therein. Applications of the variational inequalities in Contact Mechanics can be found in $[11,26,28]$. Usually, most solution methods for GVIs rely upon certain (generalized) monotonicity, convexity conditions. Recently, the convergence of regularization methods was proposed to displace (generalized) monotonicity assumptions by weak conditions, which are also sufficient for existence of solutions for GVIs in a finite dimensional space, see [12-14, 16] and references therein. Furthermore, Konnov in [13] showed that convergence of the perturbed version of the general penalty was applied to a non-stationary VI without any concordance rules and monotonicity assumptions in a finite dimensional space.

The main goal of this paper is to reveal convergence properties of the perturbed version of the regularized penalty method for GVIs in reflexive Banach spaces without any concordance rules and monotonicity assumptions for penalty and regularization parameters. At the same time, we extend those results in several directions by applying mild coercivity conditions.

\section{Preliminaries}

In this section, we recall some definitions and properties concerning nonlinear analysis, see $[1,5,6,31]$. Let $X$ be a nonempty subset of a Banach space $E$. Subsequently, the set of real numbers and the set of positive real numbers are denoted by $\mathbb{R}$ and $\mathbb{R}_{+}$, respectively. In the sequel, the symbols $x^{k} \stackrel{w}{\rightarrow} \bar{x}$ and $x^{k} \rightarrow \bar{x}$ stand for weak and strong convergence of $\left\{x^{k}\right\}$ to $\bar{x}$, respectively. Recall that the following definitions.

Definition 2.1. A function $f: X \rightarrow \mathbb{R}$ is said to be

(a) convex on a set $\mathrm{U} \subseteq X$, if for any $x, y \in U$ and $\alpha \in(0,1)$, it holds

$$
f(\alpha x+(1-\alpha) y) \leqslant \alpha f(x)+(1-\alpha) f(y) ;
$$

(b) quasiconvex on a set $\mathrm{U} \subseteq X$, if for any $x, y \in U$ and $\alpha \in(0,1)$, it holds

$$
f(\alpha x+(1-\alpha) y) \leqslant \max \{f(x), f(y)\} ;
$$

(c) explicitly quasiconvex on a set $U \subseteq X$, if it is quasiconvex and satisfies

$$
f(\alpha x+(1-\alpha) y)<\max \{f(x), f(y)\}
$$

for any $x, y \in U, x \neq y$ and $\alpha \in(0,1)$;

(d) weakly upper (lower) semicontinuous on $U \subseteq X$, if for each sequence $\left\{x^{k}\right\} \subset U$ with $x^{k} \stackrel{w}{\rightarrow} \bar{x}$, one has

$$
\limsup _{k \rightarrow \infty} f\left(x^{k}\right) \leqslant f(\bar{x})\left(\liminf _{k \rightarrow \infty} f\left(x^{k}\right) \geqslant f(\bar{x})\right) ;
$$

(e) coercive, if $\mathrm{f}(\mathrm{x}) \rightarrow+\infty$ as $\|x\| \rightarrow \infty$;

(f) weakly coercive with respect to set $X$, if there exists a constant $\rho \in \mathbb{R}$ such that the set

$$
W_{\rho}=\{x \in X \mid f(x) \leqslant \rho\}
$$

is nonempty and bounded.

Remark 2.2. Clearly, we have $(a) \Rightarrow(c) \Rightarrow(b)$ and $(e) \Rightarrow(f)$, but the reverse implications are not true in general.

Let $f:[0,1] \rightarrow \mathbb{R}$ be the function defined by $f(x)=x^{\frac{1}{2}}$, then $f$ is explicitly quasiconvex and nonconvex on $[0,1]$. Suppose that $g:[0,+\infty) \rightarrow \mathbb{R}$ is the function defined by

$$
g(x)=x^{\frac{1}{2}}, \text { if } x \in[0,1] \text { and } g(x)=1, \text { if } x \in(1,+\infty),
$$

then $g$ is quasiconvex and non-explicitly quasiconvex $[0,+\infty)$. There exists a constant $\frac{1}{2} \in \mathbb{R}$ such that the 
set

$$
W_{\frac{1}{2}}=\left\{x \in[0,+\infty) \mid g(x) \leqslant \frac{1}{2}\right\},
$$

is nonempty and bounded, yet $\mathrm{g}(\mathrm{x})=1$ as $\|x\| \rightarrow \infty$. Therefore $\mathrm{g}$ is weakly coercive with respect to the $[0,+\infty)$ and non-coercive.

If $-f$ is convex, then $f$ is called concave. Analogously, we can define the quasiconcave and explicitly quasiconcave, respectively.

Definition 2.3. We say that a family of sets $\left\{X_{k}\right\}$ is weakly Mosco convergent to a set $X$ (see [1]) if and only if

(i) for each sequence $x^{k} \in X_{k}$ with $x^{k} \stackrel{w}{\rightarrow} \bar{x}$, we have $\bar{x} \in X$;

(ii) for each point $\bar{x} \in X$, there exists a sequence $x^{k} \stackrel{w}{\rightarrow} \bar{x}$ with $x^{k} \in X_{k}$.

Next, we move our attention to the following equilibrium problem (EP, for short): find an element $x^{*} \in X$ such that

$$
\Phi\left(x^{*}, y\right) \geqslant 0, \quad \forall y \in X
$$

where $\Phi: X \times X \rightarrow \mathbb{R}$ is an equilibrium bi-function, i.e. $\Phi(x, x)=0$ for every $x \in X$.

We now give an existence result for EP (2.1) via a proper adjustment of the classical Ky Fan inequality in a Banach space, see $[8,27]$.

Proposition 2.4. Let $\mathrm{X}$ be a nonempty convex closed and bounded set in a reflexive Banach space $\mathrm{E}$, and $\Phi$ : $\mathrm{X} \times \mathrm{X} \rightarrow \mathbb{R}$ be an equilibrium bi-function such that:

(i) for each fixed $\mathrm{y} \in \mathrm{X}, \Phi(., \mathrm{y})$ is a weakly upper semicontinuous function on $\mathrm{X}$;

(ii) for each fixed $x \in X, \Phi(x,$.$) is a quasiconvex function on X$.

Then the problem EP (2.1) has a solution.

Definition 2.5. Let $X$ and $E$ be reflexive Banach spaces. A set-valued mapping $G: X \rightarrow 2^{E}$ is said to be

(g) upper semicontinuous on $X$, if for each $x \in X$ and for each open set $U$ of $E$ containing $G(x)$, there exists an open neighborhood $\mathrm{V}$ of $\mathrm{x}$ such that $\mathrm{G}(\mathrm{V}) \subseteq \mathrm{U}$;

(h) a $K($ Kakutani)-mapping on $X$, if it is upper semicontinuous on $X$ and has nonempty convex and compact values.

We consider the EP (2.1) under the following basic assumptions.

(H) Let $\mathrm{X}$ be a nonempty convex and closed set in a reflexive Banach space $E$, and $\Phi: X \times X \rightarrow \mathbb{R}$ be an equilibrium bi-function such that

(i) for each fixed $y \in X, \Phi(., y)$ is weakly upper semicontinuous;

(ii) for each fixed $x \in X, \Phi(x,$.$) is explicitly quasiconvex.$

(C) There exists a convex and lower semicontinuous function $\mu: E \rightarrow \mathbb{R}$, which is weakly coercive with respect to the set $X$, and a constant $r>0$ such that for any point $x \in X \backslash W_{r}$ where $W_{r}=\{x \in$ $X \mid \mu(x) \leqslant r\}$, there exists a point $z \in X$ with

$$
\min \{\Phi(x, z), \mu(z)-\mu(x)\}<0 \text { and } \max \{\Phi(x, z), \mu(z)-\mu(x)\} \leqslant 0 .
$$

Then, it is easy to get an existence result for EP (2.1) constrained on unbounded set by use of Proposition 2.4, see [15, Theorem 3.1].

Proposition 2.6. If $(\mathrm{H})$ and $(\mathrm{C})$ are fulfilled, then problem EP (2.1) has a solution. 
$\left(C^{\prime}\right)$ There exists a convex and lower semicontinuous function $\mu: E \rightarrow \mathbb{R}$, which is weakly coercive with respect to the set $X$, and a constant $r>0$ such that for any point $x \in X \backslash W_{r}$, there is a point $z \in \mathrm{L}_{r} \bigcap X$ such that $\Phi(x, z)<0$, where $\mathrm{L}_{r}=\{x \in X \mid \mu(x)<r\}$.

Obviously, the condition $\left(\mathrm{C}^{\prime}\right)$ implies the condition $(\mathrm{C})$. So we can get the following:

Proposition 2.7. Assume that $(\mathrm{H})$ and $\left(\mathrm{C}^{\prime}\right)$ are fulfilled, then problem EP (2.1) has a solution and all these solutions belong to $\mathrm{W}_{\mathrm{r}} \cap \mathrm{X}$.

Proof. The existence part follows directly from Proposition 2.6. It remains to verify the regularity of solution set to EP (2.1).

Arguing by contradiction, if there exists a solution $x^{\prime}$ of EP (2.1) and $x^{\prime} \notin W_{r}$, then by $\left(C^{\prime}\right)$ we have $\Phi\left(x^{\prime}, z\right)<0$ for some $z \in \mathrm{L}_{r} \bigcap \mathrm{X}$, a contradiction.

\section{Penalty method}

In this section, a general penalty method is applied to GVI (1.1) to establish the existence and convergence. We need approximation assumptions. Let $X$ be a nonempty convex and closed set in a reflexive Banach space $E$, and $G: X \rightarrow 2^{E^{*}}$ be a set-valued mapping.

$\left(A_{1}\right)$ There exists a family of nonempty convex closed subsets $\left\{X_{k}\right\}$ in $E$ which is weakly Mosco convergent to the set X.

$\left(A_{2}\right)$ There exists a family of K-mappings $G_{k}: X_{k} \rightarrow 2^{E^{*}}, k=1,2, \ldots$, such that the relations $x^{k} \stackrel{w}{\rightarrow} \bar{x}$, $y^{k} \stackrel{w}{\rightarrow} \bar{y}, x^{k} \in X_{k}, y^{k} \in X_{k}$ and $g^{k} \in G_{k}\left(x^{k}\right)$ imply $g^{k_{s}} \stackrel{w}{\rightarrow} \bar{g}$ with $\bar{g} \in G(\bar{x})$ and $\limsup _{s \rightarrow \infty}\left\langle g^{k_{s}}, y^{k_{s}}-\right.$ $\left.x^{k_{s}}\right\rangle \leqslant\langle\bar{g}, \bar{y}-\bar{x}\rangle$.

$\left(A_{3}\right)$ Let $\Psi_{k}(x, y)=\sup _{g \in G_{k}(x)}\langle g, y-x\rangle$. For each fixed $y \in X_{k}, \Psi_{k}(., y)$ is a quasiconcave functional on $X_{k}$.

We now intend to describe a general penalty method for GVI (1.1). Denote D by

$$
\mathrm{D}=\mathrm{X} \bigcap \mathrm{V},
$$

where $\mathrm{V}$ is a closed and convex set in $\mathrm{E}$. In this partition, $\mathrm{X}$ stands for a "simply" constrained set, whereas V usually includes complex or "functional" constraints. The above partition of feasible set into two subsets may be suitable for penalty method. For this reason, we suppose that $P: E \rightarrow \mathbb{R}$ is a general penalty function of $V$, i.e.,

$$
\mathrm{P}(v)=0 \text {, if } v \in \mathrm{V} \text { and } \mathrm{P}(v)>0 \text {, if } v \notin \mathrm{V} \text {. }
$$

We also introduce a family of convergence operators $\mathrm{P}_{k}$ of $\mathrm{P}$ as follows:

$\left(B_{1}\right)$ There exists a family of lower semicontinuous and convex functions $\mathrm{P}_{k}: X_{k} \rightarrow \mathbb{R}_{+}$.

$\left(\mathrm{B}_{2}\right)$ If $v^{\mathrm{k}} \in \mathrm{X}_{\mathrm{k}}, v^{\mathrm{k}} \stackrel{w}{\rightarrow} v, v \in \mathrm{X}$ and $\liminf _{\mathrm{k} \rightarrow \infty} \mathrm{P}_{\mathrm{k}}\left(v^{\mathrm{k}}\right)=0$, then $\mathrm{P}(v)=0$.

$\left(\mathrm{B}_{3}\right)$ For each point $\bar{v} \in \mathrm{D}$ there exists a sequence $v^{\mathrm{k}} \stackrel{w}{\rightarrow} \bar{v}$ with $v^{\mathrm{k}} \in \mathrm{X}_{\mathrm{k}}$ and $\mathrm{P}_{\mathrm{k}}\left(v^{\mathrm{k}}\right)=0$.

It is obvious that hypotheses $\left(B_{2}\right)$ and $\left(B_{3}\right)$ deduce the weakly Mosco convergence of functions $\left\{\mathrm{P}_{k}\right\}$ to $P$, see [1].

For each $k=1,2, \ldots$, we intend to find $\tilde{x}^{k} \in X_{k}$ and $\tilde{g}^{k} \in G_{k}\left(\tilde{x}^{k}\right)$ such that

$$
\left\langle\tilde{g}^{k}, v-\tilde{x}^{k}\right\rangle+\tau_{k}\left[P_{k}(v)-P_{k}\left(\tilde{x}^{k}\right)\right] \geqslant 0, \quad \forall v \in X_{k},
$$

where $\tau_{k}>0$ is a penalty parameter. For brevity, set

$$
\triangle_{k}(g, x, y)=\langle g, y-x\rangle+\tau_{k}\left[P_{k}(y)-P_{k}(x)\right] .
$$

Now, we turn to introduce certain coercivity conditions. For $\mu_{k}: E \rightarrow \mathbb{R}$ and a constant $\rho_{k}$ we define the level sets

$$
W \rho_{k}^{(k)}=\left\{x \in X \mid \mu_{k}(x) \leqslant \rho_{k}\right\}, \quad L \rho_{k}^{(k)}=\left\{x \in X \mid \mu_{k}(x)<\rho_{k}\right\}
$$


$\left(C_{1}\right)$ For each $k=1,2, \ldots$, there exists a convex and lower semicontinuous function $\mu_{k}: E \rightarrow \mathbb{R}$, which is weakly coercive with respect to the set $X_{k}$, and a number $\rho_{k}>0$ such that for any point $x \in$ $X_{k} \backslash W \rho_{k}^{(k)}$, there is a point $z \in L \rho_{k}^{(k)} \cap X_{k}$ such that $\triangle_{k}(g, x, z)<0$.

$\left(C_{2}\right)$ If $x^{k} \stackrel{w}{\rightarrow} \bar{x}$ and $x^{k} \in X_{k}$, then $\liminf _{k \rightarrow \infty} \mu_{k}\left(x^{k}\right) \geqslant \mu(\bar{x})$ for some $\mu: E \rightarrow \mathbb{R}$.

$\left(C_{3}\right)$ There exists a number $\theta>0$ and $\bar{v} \in \mathrm{D}$ such that for any sequences $\left\{v^{k}\right\},\left\{x^{k}\right\}$, and $\left\{g^{k}\right\}$, satisfying the conditions:

$$
v^{k} \in X_{k}, x^{k} \in X_{k}, g^{k} \in G_{k}\left(x^{k}\right),\left\|x^{k}\right\| \rightarrow+\infty, v^{k} \stackrel{w}{\rightarrow} \bar{v}
$$

it holds

$$
\liminf _{k \rightarrow \infty}\left\langle g^{k}, v^{k}-x^{k}\right\rangle \leqslant-\theta \text { and } \liminf _{k \rightarrow \infty} \mu_{k}\left(x^{k}\right) \geqslant 0
$$

$\left(C_{4}\right) \limsup _{k \rightarrow \infty} \rho_{k} \leqslant \rho^{\prime}$ for some $\rho^{\prime}>0$.

We shall prove that the sequence $\left\{x^{k}\right\}$ approximates to a solution of GVI (1.1).

Theorem 3.1. Suppose $\left(\mathrm{A}_{1}\right)-\left(\mathrm{A}_{3}\right),\left(\mathrm{B}_{1}\right)-\left(\mathrm{B}_{3}\right)$, and $\left(\mathrm{C}_{1}\right)-\left(\mathrm{C}_{4}\right)$ are fulfilled, and the sequences $\left\{\tau_{\mathrm{k}}\right\}$ satisfy

$$
\left\{\tau_{k}\right\} \nearrow+\infty \text {. }
$$

Then:

(i) GVI (3.2) has a solution for each $\tau_{k}>0$ and all these solutions belong to $W \rho_{k}^{(k)} \cap X_{k}$;

(ii) each sequence $\left\{x^{\mathrm{k}}\right\}$ of solutions of GVI (3.2) has weak limit points and all these weak limit points are solutions of GVI (1.1), which belong to $W_{\rho^{\prime}} \cap \mathrm{D}$, where $W_{\rho^{\prime}}=\left\{x \in X \mid \mu(x) \leqslant \rho^{\prime}\right\}$.

Proof. Firstly, we use hypothesis $\left(C_{1}\right)$ that for each $\tau_{k}>0,\left(C^{\prime}\right)$ is true for EP (2.1) with $\Phi(x, y)=$ $\triangle_{k}(g, x, y), X=X_{k}, \mu=\mu_{k}$ and $\rho=\rho_{k}$, thus, for any $x \in X_{k} \backslash W \rho_{k}^{(k)}$, there exists $z \in X_{k} \backslash L \rho_{k}^{(k)}$ such that

$$
\triangle_{k}(g, x, z)<0 \text {. }
$$

Since that $\Psi_{k}(., y)$ is upper semicontinuous for each $y \in X_{k}$, (see [2, Section 9.2]), therefore, for any $\lambda \in \mathbb{R}$, the set

$$
\mathrm{F}_{\lambda}=\left\{x \in X_{k} \mid \sup _{g \in G_{k}(x)}\langle g, y-x\rangle \geqslant \lambda\right\}
$$

is closed, (see [4, Proposition 1.3.4]). From hypothesis $\left(A_{3}\right)$, the convexity of $F_{\lambda}$, and reflexivity of $X$, the set $F_{\lambda}$ is weakly closed and the function $\Psi_{k}(., y)$ is weakly upper semicontinuous for each $y \in X_{k}$. Consider the function $\Phi_{k}: X_{k} \times X_{k} \rightarrow \mathbb{R}$ defined by

$$
\Phi_{k}(x, y)=\triangle_{k}(g, x, y) \text { for all } x, y \in X_{k} .
$$

Obviously, $\Phi_{k}(., y)$ is weakly upper semicontinuous for each fixed $y \in X_{k}$, but $\Phi_{k}(x,$.$) is convex for each$ fixed $x \in X_{k}$. It follows from Proposition 2.7 that GVI (3.2) has a solution $x^{k}$ with $x^{k} \in W \rho_{k}^{(k)} \cap X_{k}$, so the assertion (i) holds.

Conclusion (i) ensures that the sequence $\left\{x^{k}\right\}$ is well-defined. We are now to show that the sequence is bounded. Arguing by contradiction, without loss of generality, we suppose that $\left\|x^{k}\right\| \rightarrow+\infty$. Note that $x^{k} \in X_{k}$, besides, by $\left(B_{3}\right)$ there exists a sequence $v^{k} \stackrel{w}{\rightarrow} \bar{v}$ with $v^{k} \in X_{k}$ and $P_{k}\left(v^{k}\right)=0$. Hence, for some $g^{k} \in G_{k}\left(x^{k}\right)$, we have

$$
0 \leqslant\left\langle g^{k}, v^{k}-x^{k}\right\rangle+\tau_{k}\left[P_{k}\left(v^{k}\right)-P_{k}\left(x^{k}\right)\right]=\left\langle g^{k}, v^{k}-x^{k}\right\rangle-\tau_{k} P_{k}\left(x^{k}\right) \leqslant\left\langle g^{k}, v^{k}-x^{k}\right\rangle .
$$

Choose a subindex $\left\{k_{s}\right\}$ of $\{k\}$ such that

$$
\lim _{s \rightarrow \infty}\left\langle g^{k_{s}}, v^{k_{s}}-x^{k_{s}}\right\rangle=\liminf _{k \rightarrow \infty}\left\langle g^{k}, v^{k}-x^{k}\right\rangle
$$


We have from $\left(C_{3}\right)$,

$$
0 \leqslant \lim _{s \rightarrow \infty}\left\langle g^{k_{s}}, v^{k_{s}}-x^{k_{s}}\right\rangle \leqslant-\theta<0,
$$

which gets a contradiction. Therefore, the sequence $\left\{x^{k}\right\}$ is bounded. From the reflexivity of E, we may assume

$$
x^{k_{s}} \stackrel{w}{\longrightarrow} \bar{x}
$$

Since $x^{k} \in X_{k}$, we have $\bar{x} \in X$ by $\left(A_{1}\right)$. From $\left(C_{2}\right)$ and $\left(C_{4}\right)$ it follows that $\bar{x} \in W_{\rho^{\prime}}$. Therefore $\bar{x} \in X \cap W_{\rho^{\prime}}$.

Next, we claim that $\bar{x}$ is a solution of GVI (1.1). In fact, from (3.2) it follows that

$$
0 \leqslant \mathrm{P}_{\mathrm{k}_{\mathrm{s}}}\left(\mathrm{x}^{\mathrm{k}_{\mathrm{s}}}\right) \leqslant \tau_{\mathrm{k}_{\mathrm{s}}}^{-1}\left\langle\mathrm{~g}^{\mathrm{k}_{\mathrm{s}}}, v-\mathrm{x}^{\mathrm{k}_{\mathrm{s}}}\right\rangle+\mathrm{P}_{\mathrm{k}_{\mathrm{s}}}(v), \quad \forall v \in \mathrm{X}_{\mathrm{k}^{\prime}}
$$

where $g^{k_{s}} \in G_{k_{s}}\left(x^{k_{s}}\right)$. By $\left(B_{3}\right)$, there exists a sequence $v^{k} \stackrel{w}{\rightarrow} \bar{v}$ with $v^{k} \in X_{k}$ and $P_{k}\left(v^{k}\right)=0$ for any $\bar{v} \in$ D. Taking $v=v^{k_{s}}$ in (3.3), we obtain

$$
0 \leqslant \liminf _{s \rightarrow \infty} P_{k_{s}}\left(x^{k_{s}}\right) \leqslant \limsup _{s \rightarrow \infty}\left[\tau_{k_{s}}^{-1}\left\langle g^{k_{s}}, v^{k_{s}}-x^{k_{s}}\right\rangle\right]=0 .
$$

Hence

$$
\liminf _{s \rightarrow \infty} P_{k_{s}}\left(x^{k_{s}}\right)=0
$$

So, we have $\bar{x} \in \mathrm{V}$, i.e., $\bar{x} \in \mathrm{D}$.

Note that for any $\bar{x} \in \mathrm{D}$, there exists a sequence $v^{k} \stackrel{w}{\rightarrow} \bar{x}$ with $v^{k} \in X_{k}$ and $P_{k}\left(v^{k}\right)=0$ by $\left(B_{3}\right)$. Applying (3.2) again, for $\mathrm{g}^{\mathrm{k}_{\mathrm{s}}} \in \mathrm{G}_{\mathrm{k}_{\mathrm{s}}}\left(\mathrm{x}^{\mathrm{k}_{\mathrm{s}}}\right)$, we obtain

$$
0 \leqslant \tau_{k_{s}} P_{k_{s}}\left(x^{k_{s}}\right) \leqslant\left\langle g^{k_{s}}, v^{k_{s}}-x^{k_{s}}\right\rangle+\tau_{k_{s}} P_{k_{s}}\left(v^{k_{s}}\right) .
$$

Hence,

$$
0 \leqslant \liminf _{s \rightarrow \infty} \tau_{k_{s}} P_{k_{s}}\left(x^{k_{s}}\right) \leqslant \limsup _{s \rightarrow \infty}\left\langle g^{k_{s}}, v^{k_{s}}-x^{k_{s}}\right\rangle \leqslant\langle\bar{g}, \bar{x}-\bar{x}\rangle=0 .
$$

Therefore, we get from $\left(A_{2}\right)$ that $\lim _{s \rightarrow \infty} \tau_{k_{s}} P_{k_{s}}\left(x^{k_{s}}\right)=0$. For arbitrary $\bar{w} \in D$, using $\left(B_{3}\right)$ again, there exists a sequence $v^{k} \stackrel{w}{\rightarrow} \bar{w}$ with $v^{k} \in X_{k}$ and $P_{k}\left(v^{k}\right)=0$. For $g^{k_{s}} \in G_{k_{s}}\left(x^{k_{s}}\right)$, we have from (3.2) that

$$
\left\langle g^{k_{s}}, v^{k_{s}}-x^{k_{s}}\right\rangle-\tau_{k_{s}} P_{k_{s}}\left(x^{k_{s}}\right)=\left\langle g^{k_{s}}, v^{k_{s}}-x^{k_{s}}\right\rangle+\tau_{k_{s}}\left[P_{k_{s}}\left(v^{k_{s}}\right)-P_{k_{s}}\left(x^{k_{s}}\right)\right] \geqslant 0 .
$$

Without loss of generality, we suppose that $g^{k_{s}} \stackrel{w}{\longrightarrow} \bar{g}$, then $\bar{g} \in G(\bar{x})$. It follows that

$$
0=\lim _{s \rightarrow \infty} \tau_{k_{s}} P_{k_{s}}\left(x^{k_{s}}\right) \leqslant \limsup _{s \rightarrow \infty}\left\langle g^{k_{s}}, v^{k_{s}}-x^{k_{s}}\right\rangle \leqslant\langle\bar{g}, \bar{w}-\bar{x}\rangle .
$$

Hence $\bar{x}$ solves GVI (1.1) and assertion (ii) is true.

We note that the above proof implies that the feasible D is nonempty and GVI (1.1) and (3.1) has a solution which belongs to $W_{\rho^{\prime}} \cap D$.

Remark 3.2. If $E$ is a finite dimensional Banach space, our Theorem 3.1 will reduce to the classical one [13, Theorem 3.1].

\section{Regularized penalty method}

Now, we consider a regularized version of the penalty method for GVI (1.1), and then use a weaker coercivity condition to establish a convergence result. So, with the exception of the conditions $\left(\mathrm{C}_{1}\right)$ and $\left(C_{2}\right)$, we rely on all the assumptions of the previous section. We shall take the following.

$\left(C_{1}^{\prime}\right)$ For each $k=1,2, \ldots$, there exists a convex and lower semicontinuous function $\mu_{k}: E \rightarrow \mathbb{R}$, which 
is weakly coercive with respect to the set $X_{k}$, and a constant $\rho_{k}>0$ such that for any point $x \in$ $X_{k} \backslash W \rho_{k}^{(k)}$, there exists $z \in L \rho_{k}^{(k)} \cap X_{k}$ such that

$$
\triangle_{k}(g, x, z) \leqslant 0
$$

$\left(C_{2}^{\prime}\right)$ If $x^{k} \stackrel{w}{\rightarrow} \bar{x}$ and $x^{k} \in X^{k}$, then $\liminf _{k \rightarrow \infty} \mu_{k}\left(x^{k}\right) \geqslant \mu(\bar{x})$ for some $\mu: E \rightarrow \mathbb{R}$ and $\limsup _{k \rightarrow \infty} \mu_{k}\left(x^{k}\right) \leqslant \psi(\bar{x})$ for some $\psi: E \rightarrow \mathbb{R}$.

For each $k=1,2, \ldots$, we intend to find a point $x^{k} \in X_{k}$ such that

$$
\exists g^{k} \in G_{k}\left(x^{k}\right),\left\langle g^{k}, v-x^{k}\right\rangle+\tau_{k}\left[P_{k}(v)-P_{k}\left(x^{k}\right)\right]+\varepsilon_{k}\left[\mu_{k}(v)-\mu_{k}\left(x^{k}\right)\right] \geqslant 0, \quad \forall v \in X_{k},
$$

where $\tau_{k}>0$ and $\varepsilon_{k}>0$ are penalty parameters. For brevity, set

$$
\Phi_{k}(x, y)=\langle g, y-x\rangle+\tau_{k}\left[P_{k}(y)-P_{k}(x)\right]+\varepsilon_{k}\left[\mu_{k}(y)-\mu_{k}(x)\right] .
$$

Theorem 4.1. Suppose that $\left(\mathrm{A}_{1}\right)-\left(\mathrm{A}_{3}\right),\left(\mathrm{B}_{1}\right)-\left(\mathrm{B}_{3}\right),\left(\mathrm{C}_{1}^{\prime}\right)-\left(\mathrm{C}_{2}^{\prime}\right)$, and $\left(\mathrm{C}_{3}\right)-\left(\mathrm{C}_{4}\right)$ are fulfilled, and the sequences $\left\{\tau_{\mathrm{k}}\right\}$ and $\left\{\varepsilon_{\mathrm{k}}\right\}$ satisfy

$$
\tau_{\mathrm{k}} \nearrow+\infty, \varepsilon_{\mathrm{k}} \searrow 0 \text { as } \mathrm{k} \rightarrow \infty .
$$

Then:

(i) the solution set of GVI (4.1) is nonempty for each $\tau_{k}>0, \varepsilon_{k}>0$, and it is a subset of $W \rho_{k}^{(k)} \cap X_{k}$;

(ii) each sequence $\left\{x^{k}\right\}$ of solutions of GVI (4.1) has weak limit points and all these weak limit points are solutions of GVI (1.1), which belong to $W_{\rho^{\prime}} \cap D$, where $W_{\rho^{\prime}}=\left\{x \in X \mid \mu(x) \leqslant \rho^{\prime}\right\}$.

Proof. Firstly, we note that, for each $\tau_{k}>0$ and $\varepsilon_{k}>0,\left(C^{\prime}\right)$ is true for EP (2.1) with $\Phi(x, y)=$ $\Phi_{k}(x, y), X=X_{k}, \mu=\mu_{k}$ and $\rho=\rho_{k}$. Taking any $x \in X_{k} \backslash W \rho_{k}^{(k)}$, then there exists $y \in X_{k} \bigcap L \rho_{k}^{(k)}$ such that $\triangle_{k}(g, x, y) \leqslant 0$. It follows that

$$
\Phi_{k}(x, y)=\langle g, y-x\rangle+\tau_{k}\left[P_{k}(y)-P_{k}(x)\right]+\varepsilon_{k}\left[\mu_{k}(y)-\mu_{k}(x)\right]<0 .
$$

Since $\Psi_{k}(., y)$ is a weakly upper semicontinuous for each fixed $y \in X_{k}$, following the proof of Theorem 3.1, $\Psi_{k}(x,$.$) is a convex for each fixed x \in X_{k}$. Then so is $\Phi_{k}$, all the conditions of Proposition 2.7 hold, and GVI (4.1) has a solution. Besides, $x^{k} \in W \rho_{k}^{(k)} \cap X_{k}$ and the assertion (i) is true.

By (i), the sequence $\left\{x^{k}\right\}$ is well-defined. We have to show that it is bounded. Conversely, suppose that $\left\|x^{k}\right\| \rightarrow+\infty$. Note that $x^{k} \in X_{k}$, besides, by $\left(B_{3}\right)$ there exists a sequence $v^{k} \stackrel{w}{\rightarrow} \bar{v}$ with $v^{k} \in X_{k}$ and $P_{k}\left(v^{k}\right)=0$. Hence, for some $g^{k} \in G_{k}\left(x^{k}\right)$, we have

$$
\begin{aligned}
0 & \leqslant\left\langle g^{k}, v^{k}-x^{k}\right\rangle+\tau_{k}\left[P_{k}\left(v^{k}\right)-P_{k}\left(x^{k}\right)\right]+\varepsilon_{k}\left[\mu_{k}\left(v^{k}\right)-\mu_{k}\left(x^{k}\right)\right] \\
& =\left\langle g^{k}, v^{k}-x^{k}\right\rangle-\tau_{k} P_{k}\left(x^{k}\right)+\varepsilon_{k}\left[\mu_{k}\left(v^{k}\right)-\mu_{k}\left(x^{k}\right)\right] \\
& \leqslant\left\langle g^{k}, v^{k}-x^{k}\right\rangle+\varepsilon_{k}\left[\mu_{k}\left(v^{k}\right)-\mu_{k}\left(x^{k}\right)\right] .
\end{aligned}
$$

Note that

$$
\limsup _{k \rightarrow \infty}\left\{\varepsilon_{k}\left[\mu_{k}\left(v^{k}\right)-\mu_{k}\left(x^{k}\right)\right]\right\} \leqslant \limsup _{k \rightarrow \infty}\left[\varepsilon_{k} \mu_{k}\left(v^{k}\right)\right]-\liminf _{k \rightarrow \infty}\left[\varepsilon_{k} \mu_{k}\left(x^{k}\right)\right] \leqslant 0,
$$

on account of $\left(C_{2}^{\prime}\right)$ and $\left(C_{3}\right)$. Then, we have from $\left(C_{3}\right)$ that

$$
\begin{aligned}
0 & \leqslant \liminf _{k \rightarrow \infty}\left\{\left\langle g^{k}, v^{k}-x^{k}\right\rangle+\varepsilon_{k}\left[\mu_{k}\left(v^{k}\right)-\mu_{k}\left(x^{k}\right)\right]\right\} \\
& \leqslant \liminf _{k \rightarrow \infty}\left\langle g^{k}, v^{k}-x^{k}\right\rangle+\limsup _{k \rightarrow \infty}\left\{\varepsilon_{k}\left[\mu_{k}\left(v^{k}\right)-\mu_{k}\left(x^{k}\right)\right]\right\} \\
& \leqslant \liminf _{s \rightarrow \infty}\left\langle g^{k}, v^{k}-x^{k}\right\rangle \leqslant-\theta<0,
\end{aligned}
$$


which is a contradiction. Therefore, the sequence $\left\{x^{k}\right\}$ is bounded in the reflexive Banach space E. Without loss of generality, let $\bar{x}$ be a weak limit point of $\left\{x^{k}\right\}$, i.e.,

$$
x^{k_{s}} \stackrel{w}{\rightarrow} \bar{x} .
$$

Since $x^{k} \in X_{k}$, we have $\bar{x} \in X$ by $\left(A_{1}\right)$. From $\left(C_{2}^{\prime}\right)$ and $\left(C_{4}\right)$ it follows that $\bar{x} \in W_{\rho^{\prime}}$, therefore $\bar{x} \in X \cap W_{\rho^{\prime}}$. We claim that $\bar{x}$ is a solution of GVI (1.1), (3.1). In fact, from (4.1) it follows that

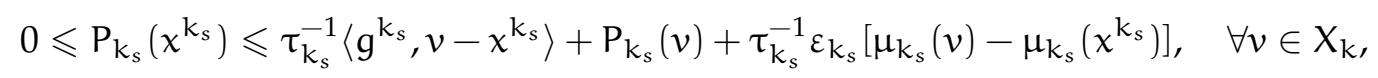

where $g^{k_{s}} \in G_{k_{s}}\left(x^{k_{s}}\right)$. By $\left(B_{3}\right)$, there exists a sequence $v^{k} \stackrel{w}{\longrightarrow} \bar{v}$ with $v^{k} \in X_{k}$ and $P_{k}\left(v^{k}\right)=0$ for any $\bar{v} \in \mathrm{D}$. Taking $v=v^{\mathrm{k}_{s}}$, we obtain

$$
0 \leqslant \liminf _{s \rightarrow \infty} P_{k_{s}}\left(x^{k_{s}}\right) \leqslant \limsup _{s \rightarrow \infty}\left[\tau_{k_{s}}^{-1}\left\langle g^{k_{s}}, v^{k_{s}}-x^{k_{s}}\right\rangle\right]+\limsup _{s \rightarrow \infty}\left\{\tau_{k_{s}}^{-1} \varepsilon_{k_{s}}\left[\mu_{k_{s}}\left(v^{k_{s}}\right)-\mu_{k_{s}}\left(x^{k_{s}}\right)\right]\right\} \leqslant 0,
$$

on account of $\left(\mathrm{A}_{2}\right),\left(\mathrm{C}_{2}^{\prime}\right)$, and (4.2), i.e.,

$$
\lim _{s \rightarrow \infty} P_{k_{s}}\left(x^{k_{s}}\right)=0,
$$

hence $\bar{x} \in \mathrm{V}$, i.e., $\bar{x} \in \mathrm{D}$. Note that there exists a sequence $v^{k} \stackrel{w}{\rightarrow} \bar{v}$ with $v^{k} \in X_{k}$ and $P_{k}\left(v^{k}\right)=0$ for any $\bar{v} \in \mathrm{D}$ due to $\left(\mathrm{B}_{3}\right)$, applying (4.1) again, for $\mathrm{g}^{\mathrm{k}_{\mathrm{s}}} \in \mathrm{G}_{\mathrm{k}_{\mathrm{s}}}\left(\mathrm{x}^{\mathrm{k}_{\mathrm{s}}}\right)$, we obtain

$$
0 \leqslant \tau_{k_{s}} P_{k_{s}}\left(x^{k_{s}}\right) \leqslant\left\langle g^{k_{s}}, v^{k_{s}}-x^{k_{s}}\right\rangle+\varepsilon_{k_{s}}\left[\mu_{k_{s}}\left(v^{k_{s}}\right)-\mu_{k_{s}}\left(x^{k_{s}}\right)\right]
$$

Hence,

$$
0 \leqslant \liminf _{s \rightarrow \infty} \tau_{k_{s}} P_{k_{s}}\left(x^{k_{s}}\right) \leqslant \limsup _{s \rightarrow \infty}\left\langle g^{k_{s}}, v^{k_{s}}-x^{k_{s}}\right\rangle+\limsup _{s \rightarrow \infty}\left\{\varepsilon_{k_{s}}\left[\mu_{k_{s}}\left(v^{k_{s}}\right)-\mu_{k_{s}}\left(x^{k_{s}}\right)\right]\right\} \leqslant\langle\bar{g}, \bar{x}-\bar{x}\rangle=0,
$$

on account of $\left(\mathrm{C}_{2}^{\prime}\right)$ and $\left(\mathrm{A}_{2}\right)$. We have

$$
\lim _{s \rightarrow \infty} \tau_{k_{s}} P_{k_{s}}\left(x^{k_{s}}\right)=0 .
$$

By $\left(\mathrm{B}_{3}\right)$, for arbitrary $\bar{w} \in \mathrm{D}$, there exists a sequence $v^{k} \stackrel{w}{\rightarrow} \bar{w}$ with $v^{k} \in X_{k}$ and $P_{k}\left(v^{k}\right)=0$. Applying (4.1) again, for $g^{k_{s}} \in G_{k_{s}}\left(\chi^{k_{s}}\right)$, we have

$$
\begin{aligned}
& \left\langle g^{k_{s}}, v^{k_{s}}-x^{k_{s}}\right\rangle-\tau_{k_{s}} P_{k_{s}}\left(x^{k_{s}}\right)+\varepsilon_{k_{s}}\left[\mu_{k_{s}}\left(v^{k_{s}}\right)-\mu_{k_{s}}\left(x^{k_{s}}\right)\right] \\
& \quad=\left\langle g^{k_{s}}, v^{k_{s}}-x^{k_{s}}\right\rangle+\tau_{k_{s}}\left[P_{k_{s}}\left(v^{k_{s}}\right)-P_{k_{s}}\left(x^{k_{s}}\right)\right]+\varepsilon_{k_{s}}\left[\mu_{k_{s}}\left(v^{k_{s}}\right)-\mu_{k_{s}}\left(x^{k_{s}}\right)\right] \geqslant 0 .
\end{aligned}
$$

Without loss of generality, we suppose that $g^{k_{s}} \stackrel{w}{\longrightarrow} \bar{g}$, then $\bar{g} \in G(\bar{x})$. It follows that

$$
0 \leqslant \lim _{s \rightarrow \infty} \tau_{k_{s}} P_{k_{s}}\left(x^{k_{s}}\right)+\liminf _{s \rightarrow \infty}\left\{\varepsilon_{k_{s}}\left[\mu_{k_{s}}\left(x^{k_{s}}\right)-\mu_{k_{s}}\left(v^{k_{s}}\right)\right]\right\} \leqslant \limsup _{s \rightarrow \infty}\left\langle g^{k_{s}}, v^{k_{s}}-x^{k_{s}}\right\rangle \leqslant\langle\bar{g}, \bar{w}-\bar{x}\rangle .
$$

Hence $\bar{x}$ solves GVI (1.1), (3.1) and assertion (ii) is true.

We observe that the above proof implies that GVI (1.1) and (3.1) has a solution under the conditions of Theorem 4.1, which are weaker than those in Theorem 3.1.

Remark 4.2. In a finite dimensional space, Theorem 4.1 somewhat generalizes the assertions of Theorem 4.1 in [13] and Theorem 2 in [14].

\section{Acknowledgment}

The work was supported by NNSF of China Grants No. 11671101, 11461021, NSF of Guangxi Grant No. 2016GXNSFBA380235, Guangxi college young and middle-aged teachers basic ability promotion project No. 2017KY0598, SF of Guangxi University of Finance and Economics Grant No. 2016D109, 2017QNA04, and Guangxi Key Laboratory Cultivation Base of Cross-border E-commerce Intelligent Information Processing and Special Funds of Guangxi Distinguished Experts Construction Engineering, Open fund of Guangxi Key laboratory of hybrid computation and IC design analysis No. HCIC201511 and Guangxi young teachers in the basic ability to enhance the project No. 2017KY0178. 


\section{References}

[1] H. Attouch, Variational convergence for functions and operators, Applicable Mathematics Series, Pitman (Advanced Publishing Program), Boston, MA, (1984). 2, 2.3, 3

[2] J. P. Aubin, Optima and equilibria, An introduction to nonlinear analysis, Translated from the French by Stephen Wilson, Second edition, Graduate Texts in Mathematics, Springer-Verlag, Berlin, (1998). 3

[3] C. Baiocchi, A. Capelo, Variational and quasivariational inequalities, Applications to free boundary problems, Translated from the Italian by Lakshmi Jayakar, A Wiley-Interscience Publication, John Wiley \& Sons, Inc., New York, (1984). 1

[4] S.-S. Chang, Variational inequalities and related problems, (Chinese) Chongqing Publishing Group, Chongqing, (2007). 3

[5] Z. Denkowski, S. Migórski, N. S. Papageorgiou, An introduction to nonlinear analysis: applications, Kluwer Academic Publishers, Boston, MA, (2003). 2

[6] Z. Denkowski, S. Migórski, N. S. Papageorgiou, An introduction to nonlinear analysis: theory, Kluwer Academic Publishers, Boston, MA, (2003). 2

[7] I. Ekeland, R. Témam, Convex analysis and variational problems, Translated from the French, Corrected reprint of the 1976 English edition, Classics in Applied Mathematics, Society for Industrial and Applied Mathematics (SIAM), Philadelphia, PA, (1999). 1

[8] K. Fan, A minimax inequality and applications, Inequalities, III (Proc. Third Sympos., Univ. California, Los Angeles, Calif., 1969; dedicated to the memory of Theodore S. Motzkin), Academic Press, New York, (1972), 103-113.. 2

[9] L. Gasiński, Z.-H. Liu, S. Migórski, A. Ochal, Z.-J. Peng, Hemivariational inequality approach to evolutionary constrained problems on star-shaped sets, J. Optim. Theory Appl., 164 (2015), 514-533. 1

[10] F. Giannessi, A. Maugeri (Ed.), Variational inequalities and network equilibrium problems, Proceedings of the conference held in Erice, June 19-25, (1994), Plenum Press, New York, (1995). 1

[11] W.-M. Han, M. Sofonea, Quasistatic contact problems in viscoelasticity and viscoplasticity, AMS/IP Studies in Advanced Mathematics, American Mathematical Society, Providence, RI; International Press, Somerville, MA, (2002). 1

[12] I. V. Konnov, Regularization method for nonmonotone equilibrium problems, J. Nonlinear. Convex. Anal., 10 (2009), 93-101. 1

[13] I. V. Konnov, Application of penalty methods to non-stationary variational inequalities, Nonlinear Anal., 92 (2013), 177-182. 1, 3.2, 4.2

[14] I. V. Konnov, An inexact penalty method for non stationary generalized variational inequalities, Set-Valued Var. Anal., 23 (2015), 239-248. 1, 4.2

[15] I. V. Konnov, Regularized penalty method for general equilibrium problems in Banach spaces, J. Optim. Theory Appl., 164 (2015), 500-513. 2

[16] I. V. Konnov, D. A. Dyabilkin, Nonmonotone equilibrium problems: coercivity conditions and weak regularization, J. Global Optim., 49 (2011), 575-587. 1

[17] Z.-H. Liu, Browder-Tikhonov regularization of non-coercive evolution hemivariational inequalities, Inverse Problems, 21 (2005), 13-20. 1

[18] Z.-H. Liu, Existence results for quasilinear parabolic hemivariational inequalities, J. Differential Equations, 244 (2008), 1395-1409.

[19] Z.-H. Liu, Anti-periodic solutions to nonlinear evolution equations, J. Funct. Anal., 258 (2010), 2026-2033.

[20] Z.-H. Liu, X.-W. Li, Approximate controllability for a class of hemivariational inequalities, Nonlinear Anal. Real World Appl., 22 (2015), 581-591.

[21] Z.-H. Liu, X.-W. Li, Approximate controllability of fractional evolution systems with Riemann-Liouville fractional derivatives, SIAM. J. Control Optim., 53 (2015), 1920-1933.

[22] Z.-H. Liu, X.-W. Li, D. Motreanu, Approximate controllability for nonlinear evolution hemivariational inequalities in Hilbert spaces, SIAM J. Control Optim., 53 (2015), 3228-3244.

[23] Z.-H. Liu, S. Migórski, S.-D. Zeng, Partial differential variational inequalities involving nonlocal boundary conditions in Banach spaces, J. Differential Equations, 263 (2017), 3989-4006.

[24] Z.-H. Liu, S.-D. Zeng, D. Motreanu, Evolutionary problems driven by variational inequalities, J. Differential Equations, 260 (2016), 6787-6799.

[25] Z.-J. Peng, Z.-H. Liu, X.-Y. Liu, Boundary hemivariational inequality problems with doubly nonlinear operators, Math. Ann., 356 (2013), 1339-1358. 1

[26] M. Sofonea, Y.-B. Xiao, Fully history-dependent quasivariational inequalities in contact mechanics, Appl. Anal., 95 (2016), 2464-2484. 1

[27] K.-K. Tan, J. Yu, X.-Z. Yuan, The stability of Ky Fan's points, Proc. Amer. Math. Soc., 123 (1995), 1511-1519. 2

[28] Y.-B. Xiao, N.-J. Huang, Y. J. Cho, A class of generalized evolution variational inequalities in Banach spaces, Appl. Math. Lett., 25 (2012), 914-920. 1

[29] Y.-B. Xiao, N.-J. Huang, J. Lu, A system of time-dependent hemivariational inequalities with Volterra integral terms, J. Optim. Theory Appl., 165 (2015), 837-853. 1 
[30] Y.-B. Xiao, X.-M. Yang, N.-J. Huang, Some equivalence results for well-posedness of hemivariational inequalities, J. Global Optim., 61 (2015), 789-802. 1

[31] E. Zeidler, Nonlinear functional analysis and its applications, II/A,B, Linear monotone operators, Translated from the German by the author and Leo F. Boron, Springer-Verlag, New York, (1990). 2

[32] S.-D. Zeng, S. Migórski, Noncoercive hyperbolic variational inequalities with applications to contact mechanics, J. Math. Anal. Appl., 455 (2017), 619-637. 1 